\title{
Positioning the Terrestrial Ephemeris Origin in the International Terrestrial Reference Frame
}

\author{
S. Lambert and C. Bizouard \\ SYRTE, UMR 8630/CNRS, Observatoire de Paris, 61 avenue de l’Observatoire, 75014 Paris, France

\begin{abstract}
Resolution B1.8 adopted by the XXIV General Assembly of the International Astronomical Union (Manchester, August 2000) recommends the use of the Non-Rotating Origin (Guinot 1979) on the moving equator both in the International Celestial Reference System (ICRS) and in the International Terrestrial Reference System (ITRS). The Non-Rotating Origin (NRO) in the ITRS is designated the Terrestrial Ephemeris Origin (TEO). Resolution B1.8 is to be implemented on 1 January 2003 by the International Earth Rotation Service (IERS) which is required to provide the position of the TEO in the ITRS. This paper is devoted to the calculation of this position. Because the TEO depends on the polar motion, which is poorly modeled, its position has been derived from observational data. This has been compared to an analytical model based on a simplified representation of polar motion. We propose then a numerical expression for the displacement of the TEO to be used in the new
\end{abstract} \\ coordinate transformation from the Celestial Reference System to the Terrestrial Reference System according to the resolution.
}

Key words. reference systems - ephemerides

\section{Introduction}

The study has been realized in the framework of the resolutions adopted by the XXIV General Assembly of the International Astronomical Union (Manchester, August 2000) concerning the transformation between the Celestial Reference System (CRS) and the Terrestrial Reference System (TRS), which are to be implemented in the International Earth Rotation Service (IERS) procedures on 1 January 2003.

Resolution B1.8 recommends the use of the Non-Rotating Origin (NRO) on the moving equator to reckon the angle of rotation of the Earth. The NRO (also called departure point) in the Celestial Reference System (CRS) is defined by the kinematical condition of non-rotation of this point around the rotation axis when the rotation pole moves in the CRS (Guinot 1979). Similarly, the NRO in the Terrestrial Reference System (TRS) is defined by the kinematical condition of non-rotation around the rotation axis when the rotation pole moves in the TRS. The properties of these two points have already been studied in Capitaine et al. (1986, 2000).

In practice, the determination of the Earth orientation refers to the Celestial Intermediate Pole (CIP), defined by IAU 2000 resolution $\mathrm{B} 1.7$, which is the pole correponding to an axis closed to the rotation axis (difference smaller than 20 mas). Therefore:

- The NRO as realized by taking into account the motion of the CIP in the Celestial Reference System (CRS) gives a point

Send offprint requests to: $\mathrm{S}$. Lambert, e-mail: Sebastien.Lambert@obspm.fr on the moving equator of date noted $\sigma$ and designated by the Celestial Ephemeris Origin (CEO).

- The NRO as realized by taking into account the motion of the CIP in the Terrestrial Reference System (TRS) gives a point on the moving equator of date noted $\varpi$ and designated as the Terrestrial Ephemeris Origin (TEO).

Astrometric and geodetic data analysis software involve the computation of the transformation between the CRS and the TRS. In the framework of the IAU 2000 recommendations, they need an expression for the displacement of the Terrestrial Ephemeris Origin both for the classical transformation and for transformation based on the Non-Rotating Origin representation.

The study is focused on the computation of the position of the TEO in the ITRS.

After having briefly recalled the expression of the quantity $s^{\prime}$ to locate the TEO (Sect. 2), we have directly computed this quantity from the observed polar motion (Sect. 3). Then a very simple analytical model of $s^{\prime}$ has been derived for a better understanding of the observed variations (Sect. 4). Finally, a numerical expression of $s^{\prime}$ is proposed for practical use in the transformation between ICRS and ITRS (Sect. 5).

\section{Formulation}

Consider a displacement of the CIP between dates $t_{0}$ and $t$, the point $\varpi$ along the moving equator defines the broken arc $s^{\prime}$ (see Fig. 1):

$s^{\prime}=\varpi M-\Pi_{0} M-\left(\varpi_{0} M_{0}-\Pi_{0} M_{0}\right)$. 


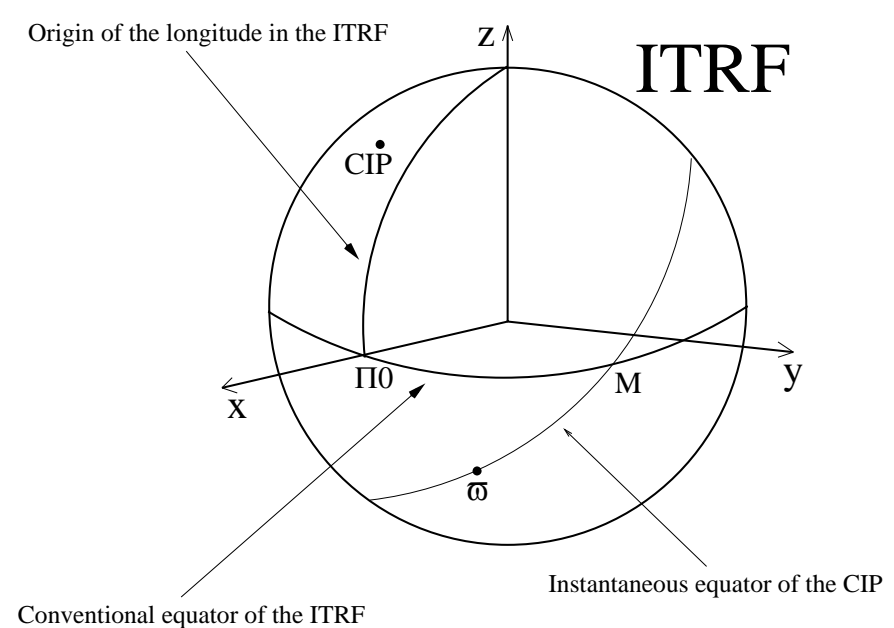

Fig. 1. The non-rotating origin with respect to the ITRF.

In this definition, $\varpi$ and $\varpi_{0}$ represent the position of the departure point at dates $t$ and $t_{0}, \Pi_{0}$ is the origin of the longitude in the ITRF, and $M$ and $M_{0}$ are the nodes between the conventional equator of the ITRF and the equators of dates $t$ and $t_{0}$. Because of the fact that the kinematical condition does not constrain the position of $\varpi_{0}$ along the moving equator, it is convenient to take, by convention (Capitaine et al. 1986):

$\varpi_{0} M_{0}-\Pi_{0} M_{0}=0$.

The displacement of the TEO in the ITRS, $s^{\prime}$, is related, for a date $t$, to the CIP coordinates $u$ and $v$ in the TRS by the formula:

$$
\begin{aligned}
s^{\prime} & =\varpi M-\Pi_{0} M \\
& =-\int_{t_{0}}^{t} \frac{u \dot{v}-\dot{u} v}{2} \mathrm{~d} t,
\end{aligned}
$$

where $u$ corresponds to the coordinate $x_{\mathrm{p}}$ of the CIP in the ITRS, $v$ refers to $-y_{\mathrm{p}}$, and the dot is for the time derivative. The integration is made between dates $t_{0}$ and $t$.

\section{Computation of the position of the TEO from geodetic data}

\subsection{Polar motion data}

The computation of $s^{\prime}$ is based upon combined time series $\mathrm{C} 04$ for polar motion which is provided by the Earth Orientation Parameters Product Center of the International Earth Rotation Service (IERS EOP-PC) based at the Paris Observatory, France. Such a series is obtained by the combination of individual polar motion series from various spatial and geodetic techniques such as Very Long Baseline radio Interferometry (VLBI), Global Positioning System (GPS, GLONASS), Satellite Laser Ranging (SLR) or Lunar Laser Ranging (LLR). This series give daily averaged values for the CIP coordinates $x_{\mathrm{p}}, y_{\mathrm{p}}$, the values for $U T 1-U T C$ and lengthof-day $L O D$, and the Celestial pole offsets $\mathrm{d} \psi$ and $\mathrm{d} \epsilon$ which are the corrections to the nutation model IAU 1980. For a sampling of one day (at $0 \mathrm{~h} U T C$ ) the data covers the period from 1962 January 1 st until today. The uncertainties on $x_{\mathrm{p}}$ and $y_{\mathrm{p}}$

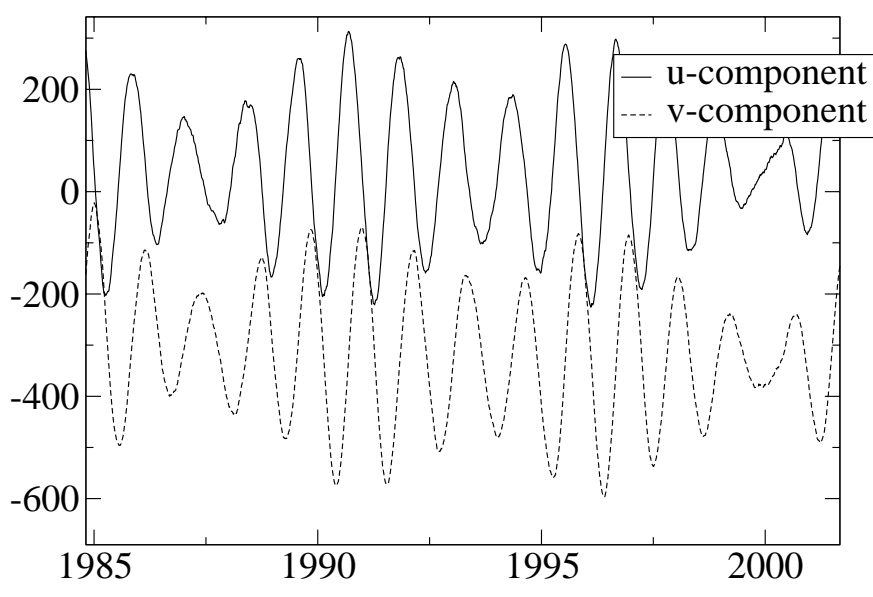

Fig. 2. Observed polar motion during the last two decades from the IERS C04 series, amplitudes are in mas, $u$-component $\left(x_{\mathrm{p}}\right)$ is in full line, $v$-component $\left(-y_{\mathrm{p}}\right)$ is in dotted line, the time is given in years.

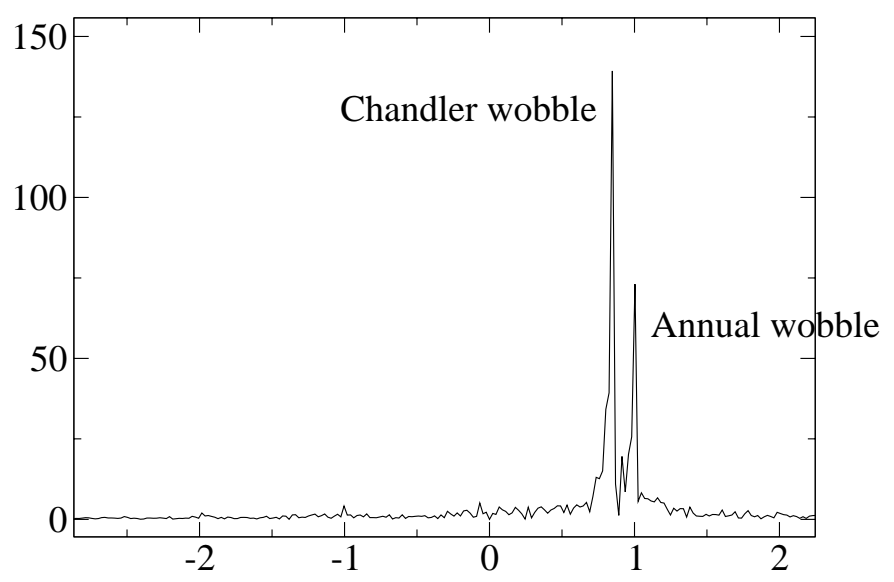

Fig. 3. Complex spectrum of polar motion (Fast Fourier Transform) over the total duration of the IERS C04 series (1962-2002), amplitudes are in mas, frequencies are in cycles per year (cpy).

as given by the IERS EOP-PC (IERS 1999) are displayed in Table 1. The most significant improvement during the early 80 's corresponds to the advent of VLBI observations in the combination. The series is plotted in Fig. 2 for the two coordinates and a FFT spectrum of these coordinates is shown in Fig. 3.

The GPS technique allows us to estimate directly the rates $\dot{x}_{\mathrm{p}}$ and $\dot{y}_{\mathrm{p}}$. Analysis Centers providing series of polar motion rates are mainly the Center for Orbit Determination in Europe (CODE) since 1993.5, the Jet Propulsion Laboratory (JPL, USA) since 1992.4 and the GeoForschungsZentrum (GFZ, Germany) since 1996.5 .

The computation of $s^{\prime}$ has been performed by using pole coordinates $x_{\mathrm{p}}$ and $y_{\mathrm{p}}$ of the C04 series (Sect. 3.2) and their rates $\dot{x}_{\mathrm{p}}$ and $\dot{y}_{\mathrm{p}}$, and by using the pole coordinates rates from the GPS series (Sect. 3.3). 
Table 1. Uncertainty of a daily value (in mas) on the IERS C04 Earth Orientation Parameters series.

\begin{tabular}{ccccccc}
\hline \hline & $62-67$ & $68-71$ & $72-79$ & $80-83$ & $84-95$ & $95-02$ \\
\hline$x_{\mathrm{p}}$ & 30 & 20 & 15 & 2 & 0.7 & 0.2 \\
$y_{\mathrm{p}}$ & 30 & 20 & 15 & 2 & 0.7 & 0.2 \\
\hline
\end{tabular}

\subsection{Processing using CO4 data}

Let $u=x_{\mathrm{p}}$ and $v=-y_{\mathrm{p}}$ be the CIP coordinates in the ITRS. From time series of $u$ and $v$, we can compute numerically the quantity $s^{\prime}$ given by Eq. (3).

First, a numerical derivative of $u$ and $v$ has been applied by the following algorithm. For a numerical series $S$ whose sampling is based on a time scale $T$, the numerical derivative $D$ at the point number $n$ is given by:

$D_{n}=\dot{S}_{n}=\frac{S_{n+1}-S_{n-1}}{T_{n+1}-T_{n-1}}$.

For the time integral, we have used a basic trapezoid method. The relationship between the series $S$ and its time integral $I$ is:

$I_{n}=I_{n-1}+\frac{S_{n}+S_{n-1}}{2}\left(T_{n}-T_{n-1}\right)$,

with $I_{0}=0$. The result of the computation is plotted in Fig. 4 . It can be noted that the 30 mas error in data given in Table 1 leads to an error in $s^{\prime}$ smaller than $0.01 \mu$ as.

We notice that the curve of $s^{\prime}$ shows a periodic variation with period 2334.4 days with an amplitude that reaches $1 \mu$ as and which is also present in the polar motion (see Fig. 2). Smaller oscillations with Chandlerian and annual periods are also visible. The trend of the curve is varies during the 40 years depending on the variations of the amplitudes of the chandlerian and annual wobbles. It is a well known fact that the Chandler wobble has undergone some drastic variations in amplitude and phase during the twentieth century. For details see Guinot (1972) and Vondrák (1985).

\subsection{Processing using GPS data}

Another computation has been made using direct estimates of the rates $\dot{u}$ and $\dot{v}$ from GPS observations. The use of these estimates is only possible for recent observations, but it provides an independant computation which can be compared with the previous results during the common interval. Differences between $s^{\prime}$ computed with the numerical derivative using C04 series and $s^{\prime}$ computed with the estimated rates using various GPS data are plotted in Fig. 5. These differences, which never reach more than $0.1 \mu$ as, are not significant at the present level of EOP accuracy. This validates the two independant methods of computation.

\section{Computation of the position of the TEO based on model for polar motion}

\subsection{Low-frequency polar motion}

It is well known that the polar motion, at low frequencies (periods longer than a few days), is mostly a combination of the

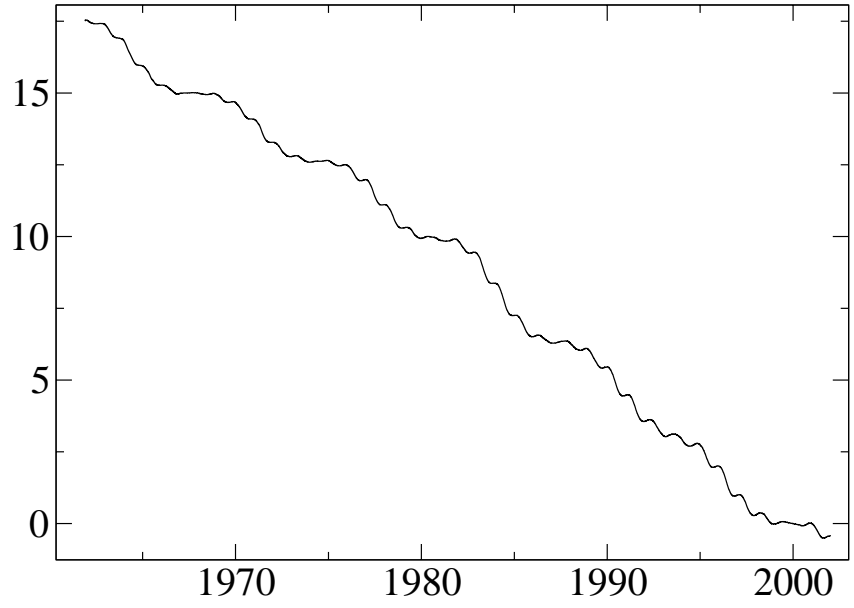

Fig. 4. Displacement $s^{\prime}$ of the Terrestrial Ephemeris Origin over the total duration of the IERS C04 series (1962-2002), the time is given in years, amplitudes are in $\mu$ as.

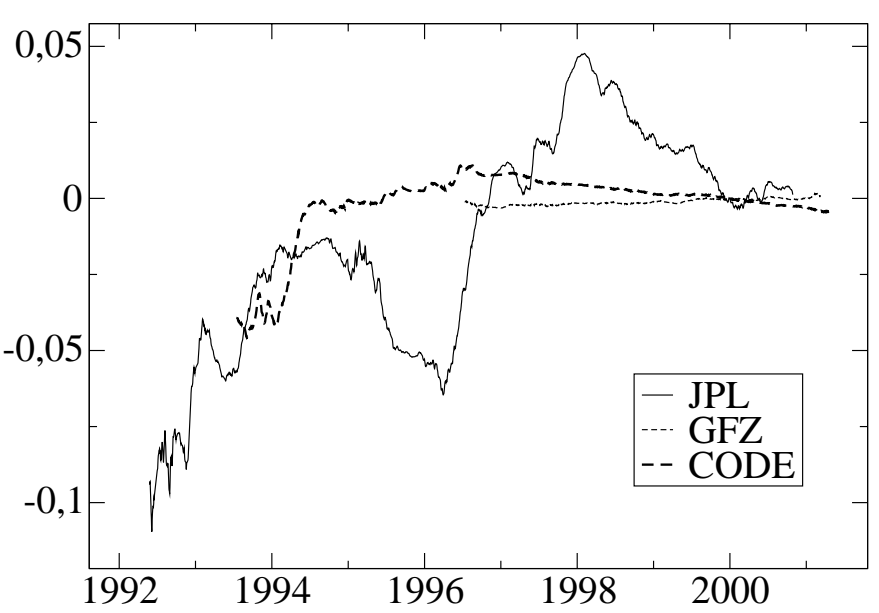

Fig. 5. Differences between $s^{\prime}$ computed from C04 data and $s^{\prime}$ computed from various GPS data sets using estimated pole coordinates rate, the time is in years, amplitudes are in $\mu$ as.

Chandler wobble, with a period of about 433 days (Lambeck 1988), and the annual oscillation. The Fourier spectrum in Fig. 3 shows the two main peaks at 433 days and 365.25 days. The combination of these two oscillations causes a modulation of 2334.4 days (about 6.4 years) which is clearly visible in Fig. 2. In addition, we observe a linear trend in both coordinates.

In this section, we assume that the observed polar motion is well represented by two prograde circular waves at the above periods and by a linear trend:

$u=A_{\mathrm{c}} \cos \left(\sigma_{\mathrm{c}} t+\phi_{\mathrm{c}}\right)+A_{\mathrm{a}} \cos \left(\sigma_{\mathrm{a}} t+\phi_{\mathrm{a}}\right)+u_{0}+u_{1} t$,
$v=A_{\mathrm{c}} \sin \left(\sigma_{\mathrm{c}} t+\phi_{\mathrm{c}}\right)+A_{\mathrm{a}} \sin \left(\sigma_{\mathrm{a}} t+\phi_{\mathrm{a}}\right)+v_{0}+v_{1} t$,

where $A_{\mathrm{c}}$ and $A_{\mathrm{a}}$ are the real amplitudes of the Chandler and annual wobbles respectively and are considered as constant over the span of the series, $\sigma_{\mathrm{c}}$ and $\sigma_{\mathrm{a}}$ are the Chandler and annual frequencies, with the respective phases $\phi_{\mathrm{c}}$ and $\phi_{\mathrm{a}}$ refered to epoch J2000.0. The non-periodic terms are represented by $u_{0}$, 
Table 2. Least-squares fitting of the Chandler-Annual model using the IERS C04 series over three periods of twenty years.

\begin{tabular}{lrrr}
\hline \hline Parameter & $1962-1982$ & $1972-1992$ & $1982-2002$ \\
\hline$A_{\mathrm{c}}$ (mas) & 140 & 160 & 178 \\
$A_{\mathrm{a}}$ (mas) & 92 & 87 & 80 \\
\hline$\phi_{\mathrm{c}}$ (degrees) & -73 & -59 & -58 \\
$\phi_{\mathrm{a}}$ (degrees) & 107 & 110 & 120 \\
\hline$u_{0}$ mas & 68 & 63 & 41 \\
$u_{1}$ mas per mjd & 0.006 & 0.005 & 0.000 \\
$v_{0}$ mas & -378 & -364 & -341 \\
$v_{1}$ mas per mjd & -0.014 & -0.013 & -0.009 \\
\hline
\end{tabular}

$v_{0}, u_{1}$ and $v_{1}$. It is easy to write then

$\dot{u}=-\sigma_{\mathrm{c}} A_{\mathrm{c}} \sin \left(\sigma_{\mathrm{c}} t+\phi_{\mathrm{c}}\right)-\sigma_{\mathrm{a}} A_{\mathrm{a}} \sin \left(\sigma_{\mathrm{a}} t+\phi_{\mathrm{a}}\right)+u_{1}$,

$\dot{v}=\sigma_{\mathrm{c}} A_{\mathrm{c}} \cos \left(\sigma_{\mathrm{c}} t+\phi_{\mathrm{c}}\right)+\sigma_{\mathrm{a}} A_{\mathrm{a}} \cos \left(\sigma_{\mathrm{a}} t+\phi_{\mathrm{a}}\right)+v_{1}$.

After an analytical integration, we obtain a model for $s^{\prime}$ in the form:

$s^{\prime}(t)=L(t)+B(t)+P(t)$,

where the first term is the linear trend

$L(t)=-\frac{1}{2}\left[\sigma_{\mathrm{c}} A_{\mathrm{c}}^{2}+\sigma_{\mathrm{a}} A_{\mathrm{a}}^{2}+v_{1} u_{0}-u_{1} v_{0}\right] t$.

The second term contains periodic terms of period 2334.4 days:

$B(t)=-\frac{1}{2} \frac{\sigma_{\mathrm{c}}+\sigma_{\mathrm{a}}}{\sigma_{\mathrm{c}}-\sigma_{\mathrm{a}}} A_{\mathrm{c}} A_{\mathrm{a}} \sin \left(\left(\sigma_{\mathrm{c}}-\sigma_{\mathrm{a}}\right) t+\left(\phi_{\mathrm{c}}-\phi_{\mathrm{a}}\right)\right)$.

The last term is less significant and more complex. It contains Chandler and annual periods and combined terms $t \times \sin$ or $t \times \cos$ :

$$
\begin{aligned}
P(t)= & -\left[\frac{u_{1}}{\sigma_{\mathrm{c}}}-\frac{1}{2}\left(v_{0}+v_{1} t\right)\right] A_{\mathrm{c}} \cos \left(\sigma_{\mathrm{c}} t+\phi_{\mathrm{c}}\right) \\
& -\left[\frac{v_{1}}{\sigma_{\mathrm{c}}}+\frac{1}{2}\left(u_{0}+u_{1} t\right)\right] A_{\mathrm{c}} \sin \left(\sigma_{\mathrm{c}} t+\phi_{\mathrm{c}}\right) \\
& -\left[\frac{u_{1}}{\sigma_{\mathrm{a}}}-\frac{1}{2}\left(v_{0}+v_{1} t\right)\right] A_{\mathrm{a}} \cos \left(\sigma_{\mathrm{a}} t+\phi_{\mathrm{a}}\right) \\
& -\left[\frac{v_{1}}{\sigma_{\mathrm{a}}}+\frac{1}{2}\left(u_{0}+u_{1} t\right)\right] A_{\mathrm{a}} \sin \left(\sigma_{\mathrm{a}} t+\phi_{\mathrm{a}}\right) .
\end{aligned}
$$

As we mentionned above, Chandlerian and annual wobbles amplitudes have known variations. To illustrate these variations, least-squares fits of Chandler, annual and linear terms over three windows spanning twenty years were performed. Note that the data span has to be greater than the modulation period of 433-day and 365.25-day wobbles, i.e. 6.4 years for their decorellation. Table 2 displays the results. Over the complete span 1962-2002, the estimates gives $A_{\mathrm{c}}=158$ mas, $A_{\mathrm{a}}=$ 85 mas, $\phi_{\mathrm{c}}=-64^{\circ}, \phi_{\mathrm{a}}=113^{\circ}, u_{0}=52 \mathrm{mas}, v_{0}=-348 \mathrm{mas}$, $u_{1}=0.004$ mas per century and $v_{1}=-0.012$ mas per century. The quantity $s^{\prime}$ associated with this model has been computed from Eq. (8). It is compared to the previous results from
Table 3. Size of terms of $s^{\prime}$ as presented in Eqs. (8)-(10).

\begin{tabular}{lr}
\hline \hline$\sigma_{\mathrm{c}} A_{\mathrm{c}}^{2}$ & $100 \mu \mathrm{as} / \mathrm{c}$ \\
$\sigma_{\mathrm{a}} A_{\mathrm{a}}^{2}$ & $10 \mu \mathrm{as} / \mathrm{c}$ \\
$u_{1} v_{0}$ & $10^{-6} \mu \mathrm{as} / \mathrm{c}$ \\
$v_{1} u_{0}$ & $10^{-6} \mu \mathrm{as} / \mathrm{c}$ \\
$\frac{\sigma_{\mathrm{c}}+\sigma_{\mathrm{a}}}{\sigma_{\mathrm{c}}-\sigma_{\mathrm{a}}} A_{\mathrm{c}} A_{\mathrm{a}}$ & $1 \mu \mathrm{as}$ \\
Other terms in $P$ & $10^{-8} \mu \mathrm{as}$ \\
\hline
\end{tabular}

Table 4. Values of the main terms of $s^{\prime}$ as presented in Eqs. (8)-(10) for three different periods.

\begin{tabular}{lrrr}
\hline \hline & $1962-1982$ & $1972-1992$ & $1982-2002$ \\
\hline$L \mu \mathrm{as} / \mathrm{c}$ & -38 & -44 & -51 \\
$B \mu \mathrm{as}$ & 0.4 & 0.4 & 0.4 \\
\hline
\end{tabular}

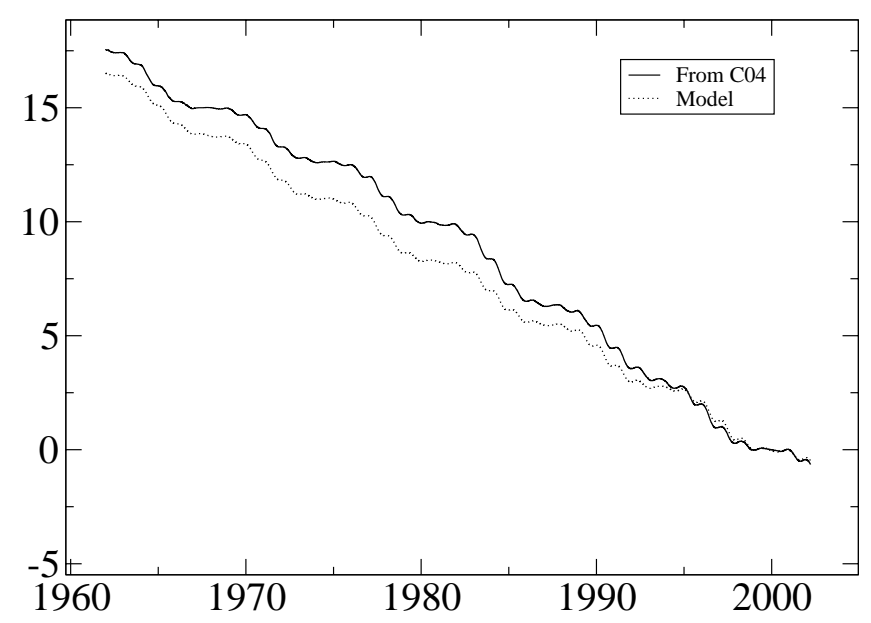

Fig. 6. Quantity $s^{\prime}$ computed from observational data (full line) and from an analytical model (dotted line).

C04 data in Fig. 6. The deviation is caused by changes in amplitudes of the wobbles and the changes in the trends.

Now, let us evaluate the size of each term in view of having an accuracy of $1 \mu$ as over a century. We take the typical values of $A_{\mathrm{c}}=200$ mas, $A_{\mathrm{a}}=100$ mas, $u_{0}=50$ mas, $v_{0}=-400$ mas, $u_{1}=0.005$ mas per Julian century and $v_{1}=100$ mas per Julian century. It leads to the values diplayed in Table 3 . We notice that the periodic term coming from $B(t)$ is near the level of accuracy desired and other periodic terms $P(t)$ are totally negligible, as well as secular terms $u_{0} v_{1}$ and $u_{1} v_{0}$. Considering the least-squares estimates reported in Table 2, we have computed the amplitudes of the main terms expressed in above equations, $L$ and $B$, for three periods of 20 years. Results are displayed in Table 4 . The periodic term $B(t)$ is, with these values, below the desirable accuracy, and should be neglected. At $1 \mu$ as accuracy over the last 40 years, the expression for $s^{\prime}$ is reduced to a linear form:

$s^{\prime}(t)-s^{\prime}\left(t_{0}\right)=-\frac{1}{2}\left[\sigma_{\mathrm{c}} A_{\mathrm{c}}^{2}+\sigma_{\mathrm{a}} A_{\mathrm{a}}^{2}\right] t$. 


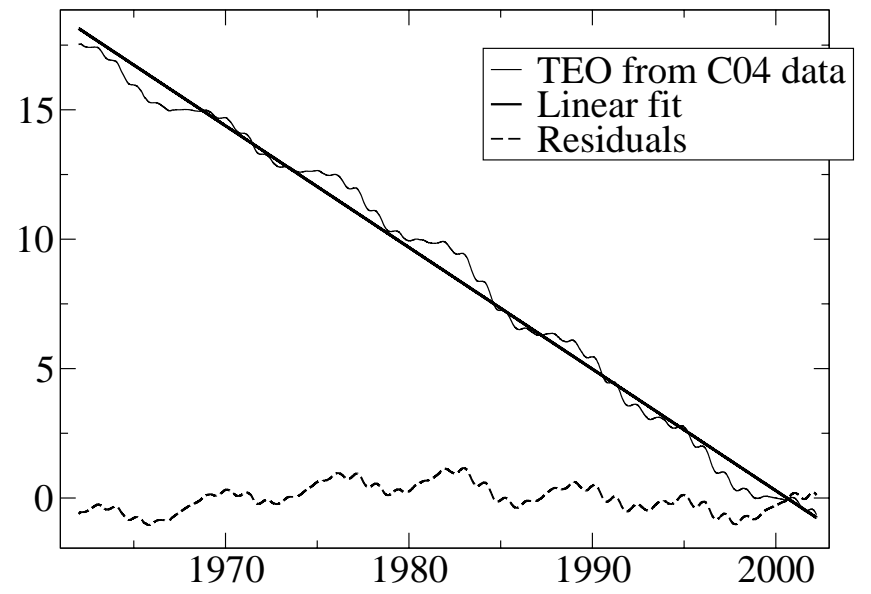

Fig. 7. Displacement of the Terrestrial Ephemeris Origin $s^{\prime}$ over the total duration of the IERS C04 series (1962-2000) and the model given by expression 15 . The time is given in years, displacements are in $\mu$ as.

\subsection{High-frequency polar motion}

The diurnal and subdiurnal tidal variations for polar motion provided by Ray (McCarthy 1996; Ray 1994) should be added for a more rigourous study due to possible effects from their very short periods in the time integration. These highfrequency contributions are very small compared to the amplitude of the long periodic polar motion: they can reach a maximum of 0.5 mas.

We have computed analytically the contribution of these corrections $\delta u$ and $\delta v$ in $s^{\prime}$. The analytical model used in the IERS Conventions (McCarthy 1996) is in the form:

$$
\begin{gathered}
\delta u=\sum_{i=1}^{8}\left(A_{\mathrm{s}, i}^{u} \sin \left(\sigma_{i} t\right)+A_{\mathrm{c}, i}^{u} \cos \left(\sigma_{i} t\right)\right), \\
\delta v=\sum_{i=1}^{8}\left(A_{\mathrm{s}, i}^{v} \sin \left(\sigma_{i} t\right)+A_{\mathrm{c}, i}^{v} \cos \left(\sigma_{i} t\right)\right) .
\end{gathered}
$$

The secular contribution in $s^{\prime}$ deduced from this model is, after a short calculation similar to the one done in the preceding paragraph:

$s^{\prime}(t)=-\frac{1}{2} \sum_{i=1}^{8} \sigma_{i}\left(A_{\mathrm{c}, i}^{u} A_{\mathrm{s}, i}^{v}+A_{\mathrm{s}, i}^{u} A_{\mathrm{c}, i}^{v}\right) t$.

The effect is only about $0.06 \mu$ as per century and is therefore negligible.

\section{Expression for positioning the TEO in the ITRF}

As a conclusion of this study, we propose a numerical expression for the quantity $s^{\prime}$. Although such a model is limited in time because of unpredictable changes in the wobbles amplitudes, it is possible to provide a numerical expression for $s^{\prime}$ which represents the motion of the TEO in the ITRS with an accuracy of $1 \mu$ as over the last 40 years. On this span, variations in the amplitudes of the wobbles will not produce an error larger than $1 \mu$ as. The following expression is fitted on the curve obtained from $\mathrm{C} 04$ data:

$s^{\prime}=-47.0 \times t$,

where $s^{\prime}$ is in $\mu$ as. The parameter $t$ is the Terrestrial Time (TT) expressed in julian centuries from epoch J2000.0:

$t=(\mathrm{TT}-2000$ January 1d 12h TT)/36 525,

with TT in days.

If the Chandler amplitude in the next years does not present variations larger than during the span 1962-2002, then according to Table 4, the uncertainty on the linear trend is $13 \mu$ as per julian century. We can ensure that the $1 \mu$ as accuracy linear model can be extended for the 10 next years.

Figure 7 shows the linear model of $s^{\prime}$ corresponding to expression (15) together with the numerical computation from observations. If unexpected variations in the amplitudes of the wobbles occur in the coming years, such a model will have to be updated.

\section{References}

Capitaine, N., Guinot, B., \& Souchay, J. 1986, Celest. Mech., 39, 283 Capitaine, N., Guinot, B., \& McCarthy, D. D. 2000, A\&A, 355, 398

Capitaine, N., Chapront, J., Lambert, S., \& Wallace, P. 2002, A\&A, submitted

Guinot, B. 1972, A\&A, 19, 207

Guinot, B. 1979, in Time and the Earth's Rotation, ed. D. D. McCarthy, \& J. D. Pilkington (D. Reidel Pub. Co.), 7

IERS 1999, IERS annual report 1999, Observatoire de Paris

Lambeck, K. 1988, Geophysical Geodesy, The Slow Deformation of the Earth (Oxford Science Publications)

McCarthy, D. D. 1996, IERS Conventions, IERS Technical Note 21 (Observatoire de Paris)

Ray, R., Steinberg, D. J., Chao, B. F., \& Cartwright, D. E. 1994, Science, 264, 830

Vondrák, J. 1985, Ann. Geophys., 3, 351 